\title{
EVALUATION OF CELLULAR PREDICTION MODELS USING 900 MHz OUTDOOR MEASUREMENTS AND TUNING OF LEE MODEL OVER INDIAN URBAN \& SUBURBAN REGIONS
}

M V S N Prasad ${ }^{1}$, K Ratnamala ${ }^{2}$, P K Dalela ${ }^{3}$, Chandra Shekhar Misra ${ }^{4}$,

1. National Physical Laboratory, Dr. K S Krishnan road, New Delhi, India, e-mail: mvsnprasad@gmail.com, mvprasad@mail.nplindia.org

2. National Geophysical Research Institute, Hyderabad, India, email: kunala ratnamala@rediffmail.com

3. C-DOT, Chattarpur, Mehraulli, New Delhi, India, email: pdalela@gmail.com

4. Aircom International Pvt Ltd., Gurgaon, India, email: chandra.misra@aircom.co.uk

\begin{abstract}
:
The present study reports the field strength measurements of some GSM transmitters in the $900 \mathrm{MHz}$ band located in the urban and suburban regions of Delhi in India. The measured signal levels converted into path loss values have been compared with the losses predicted from models like Hata, Lee and COST 231 Walfisch \& Ikegami. The prediction errors and standard deviations of the predictions errors have been deduced. Based on these results, Lee prediction method has been tuned and new model parameters have been derived. The model comparison is done in terms of statistical parameters like, root mean square error, coefficient of determination and average hit rate error.

Key words: $900 \mathrm{MHz}$ measurements, path loss modeling, Lee model tuning

\section{Introduction}

Due to the rapid developments in cellular communication over this country and the pace with which the cellular data base is increasing, the standard 900 and $1800 \mathrm{MHz}$ spectral bands are becoming increasingly congested with the demands of more frequency allocation by many operators. An efficient spectral allocation requires the testing of
\end{abstract}


various prediction methods. The evaluation of the prediction methods also helps to determine the interference levels and help in electromagnetic interference and compatibility studies. The $3 \mathrm{G}$ communication systems ready to be launched at any time in the country also require good field trials and identification of coverage prediction methods. In the past measurements in the $800 / 900 \mathrm{MHz}$ band have been reported by workers [1] and they tried to explain the extensive field results through theoretical analysis and modeling of the physical phenomena. Some of the well known mobile operators utilized calibrated statistical models for coverage and planning purposes derived from Okumara-Hata model [2-3]. Vieira et al [4] extensively discussed the refarming of $900 \mathrm{MHz}$ band into HSPA and LTE and stressed the importance of this band for rural mobile broadband applications. The Lee propagation model has been recognized by the wireless industry as one of the most accurate propagation model in the $900 \mathrm{MHz}$ band [5] wherein the authors Lee \& Lee discussed several innovative and revolutionary approaches that can better handle issues created by rough terrain sampling data.

The special feature in Delhi urban environment is most of the buildings are non uniformly spaced and results reported elsewhere might not be totally applicable to this type of environment. With this background and to evaluate the suitability of the existing models, field strength measurements were conducted in the $900 \mathrm{MHz}$ band utilizing the following GSM base stations situated in national capital region of Delhi. They are 1.Paschimvihar (PVR) 2. University Area (UA) 3. Nandanagari -1 (NN-1). 4. Nandanagari- 2 (NN-2) 5. Satyaniketan (SNT) 6. Faridabad (FBD) 7. Vinayak Hospital (VKH) and three suburban base stations namely 1. Tradex tower (TXT), 2. Meethapur (MPR) 3. Gurgaon (GRN). All the base stations shown on google earth map 
are in figure 1 and figures $2 \mathrm{a}-2 \mathrm{c}$ show the map depicting the measurement route for three base stations. These have been incorporated to get a feel of the environment for the reader. The measured values of signal strength have been compared with the following prediction methods 1. Hata [4] 2. Lee [5-6] 3. COST 231 Walfisch \& Ikegami [7]. The models have been chosen so that they are applicable to the environments where measurements were conducted. An attempt is also made to tune the Lee method based on the measured values. This type of work is the first of its kind from this region of the world.

\section{Environmental descriptions}

Both the NN-1 and NN-2 base stations are surrounded by dense urban areas on three sides and medium urban environment on the other side. The average building height is around 6 to $9 \mathrm{~m}$. PVR base station is surrounded by medium urban environment with slight open areas in between. Far away from the base station on the left side low tree density is seen. On the southern side of the base station a small water canal flows. UA base station is surrounded by medium urban environment and from east to south of the base station a thick patch of tree density is seen. At distances $2 \mathrm{~km}$ away from the base station dense urban environment prevails on the southern side of the base station. MPR base station is surrounded by low density urban area (mainly suburban) and small patches

of green vegetation in between. SNT base station is surrounded by medium urban environment and small patches of greenery in between denoting some kind of low urban residential environment. Clutter features of NN-1 and UA base station are shown in figures 3 and 4 to get a glimpse of measurement sites.

\section{Experimental details}


The specifications are shown in Table 1 . In table 1 after the base station acronyms, the transmitting power in $\mathrm{dBm}$ and transmitting antenna gain in $\mathrm{dBi}$ are shown in brackets. $\mathrm{H}_{\mathrm{b}}$ denotes the height of the base station antenna above ground. The receiver is standard Nokia equipment used in drive in tools for field trials. The position of the mobile is determined from the GPS receiver and this information with the co-ordinates of the base station was utilized to deduce the distance traveled by the mobile from the base station. The signal strength information recorded in $\mathrm{dBm}$ was converted into path loss values utilizing the gains of the antenna. Field strength samples recorded along a route must be properly managed to obtain statistics that represent accurate mean values of received field strength level. The Lee method [10-11] is the reference technique to determine the local mean values of the signal measured in movement. This technique is recommended by International organizations ITU-R[12 ] and CEPT[13 ]. A generalized method for a wider use was defined in [14 ]. Both methods define three parameters to be considered for an accurate estimation of field strength mean values along a route: the minimum number of equally-spaced samples that must be considered in the average, the minimum distance between uncorrelated samples and the appropriate distance that must be used in the sample averaging. The data was recorded with 512 samples in one second on a laptop and the number of samples collected in the present study varied from $1 \times 10^{5}$ to $2 \times 10^{5}$. Hence all the samples provide representative field strength mean value. Measured r.m.s. error is $1.5 \mathrm{~dB}$. Data was averaged over conventional figure of $40 \lambda$.

\section{Methodology of study}

In the present study observed field strength values have been converted into path loss values as a function of distance and compared with various empirical models like Hata, Lee and COST 231 Walfisch \& Ikegami. The comparison is shown in figures for some typical base stations in the 
section on results. From this comparison deviation of the models have been deduced. Also from the observed values of path loss values, path loss exponents have been deduced as a function of distance. From the prediction errors statistical parameters like RMSE, $\mathrm{R}^{2}$ and AHRE have been deduced to evaluate the suitability of the models. These are described in detail in the subsequent sections. Based on these results an attempt is made to tune the Lee method and methodology of tuning along with the discussion of tuned results has been presented. The statistical parameters of tuned Lee method have been compared with Hata and COST 231 Walfisch-Ikegami methods.

Also brief description of the models is shown below

4.1 Brief description of the prediction models: The three models used in this study are briefly

described. Complete details can be had from the references.

Hata Method: Okumara's prediction of median path loss[8] are usually calculated using

Hata's approximations as follows[6].

$$
\begin{aligned}
\mathrm{L}(\mathrm{dB}) & =\mathrm{A}+\mathrm{B} \log \mathrm{R}-\mathrm{E} \text { for urban areas } \\
& =\mathrm{A}+\mathrm{B} \log \mathrm{R}-\mathrm{C} \text { for suburban areas } \\
& =\mathrm{A}+\mathrm{B} \log \mathrm{R}-\mathrm{D} \text { for open areas }
\end{aligned}
$$

Where $A=69.55+26.16 \log f-13.82 \log h_{b}$,

$$
B=44.9-6.55 \log h_{b}, \quad C=2\left(\log \left(f_{c} / 28\right)\right)^{2}+5.4
$$$$
D=4.78\left(\log f_{c}\right)^{2}+18.33 \log f_{c}+40.94
$$$$
E=3.2\left(\log \left(11.75 h_{m}\right)\right)^{2}-4.97 \text { for large cities, } f_{c} \geq 300 \mathrm{MHz}
$$$$
\mathrm{E}=8.29\left(\log \left(1.54 \mathrm{~h}_{\mathrm{m}}\right)\right)^{2}-1.1 \text { for large cities, } \mathrm{f}_{\mathrm{c}}<300 \mathrm{MHz}
$$$$
E=\left(1.1 * \log \left(f_{c}\right)-0.7\right) h_{m}-\left(1.56 * \log \left(f_{c}\right)-0.8\right) \text { for medium to small cities }
$$ 
$\mathrm{f}=$ Operating frequency in $\mathrm{MHz}, \mathrm{h}_{\mathrm{b}}=$ Effective height of Transmitting antenna in meter $\mathrm{h}_{\mathrm{m}}=$ Effective height of mobile antenna in meters, $\mathrm{R}=$ distance from transmitter in $\mathrm{km}$. The model is used for distances starting from one km onwards.

Lee Method: The Lee area to area model is given by

$$
\mathrm{L}(\mathrm{dB})=\mathrm{L}_{0}+\gamma \log (\mathrm{d})-10 \log \left(\mathrm{F}_{0}\right)
$$

where $\mathrm{L}$ represents the path loss value at a given distance, $\mathrm{L}_{0}$ is a median path loss at 1 $\mathrm{km}$ distance, $\gamma$ is the slope of the path loss curve and $\mathrm{F}_{0}$ is the adjustment factor comprised of several factors, $F_{0}=F_{1} F_{2} F_{3} F_{4} F_{5}$ which have been explained in the original Lee papers.

\section{COST 231 WALFISCH-IKEGAMI METHOD}

In the non-LOS case the basic transmission loss comprises the free space path loss $L_{B}$ the multiple screen diffraction loss $\mathrm{L}_{\mathrm{msd}}$ and the rooftop to street diffraction and scatter loss $\mathrm{L}_{\mathrm{rts}}$. Thus the path loss $\mathrm{L}_{\mathrm{b}}$ in non LOS is defined as

$$
L_{b}=\left\{\begin{array}{lr}
L_{B}+L_{r t s}+L_{m s d} & L_{r t s}+L_{m s d}>0 \\
L_{B} & L_{r t s}+L_{m s d}<0
\end{array}\right.
$$

The determination of $\mathrm{L}_{\mathrm{rts}}$ is based on the principle given in the Ikegami model, but with a different street orientation function. The values of $\mathrm{L}_{\mathrm{rts}}$ are as follows

$$
L_{r t s}=-16.9-10 \log _{10} w+10 \log _{10} f_{M H z}+20 \log _{10}\left(h-h_{m}\right)+L_{\text {ori }}
$$

where $\mathrm{w}, \mathrm{h}$ and $\mathrm{h}_{\mathrm{m}}$ are gap between buildings, height of building and height of mobile stations respectively.

$$
L_{\text {ori }}=\left[\begin{array}{lc}
-10+0.354 \psi & 0^{0} \leq \psi<35^{0} \\
2.5+0.075(\psi-35) & 35^{0} \leq \psi<55^{0} \\
4.0-0.114(\psi-55) & 55^{0} \leq \psi<90^{0}
\end{array}\right.
$$

where $\mathrm{L}_{\text {ori }}$ is a factor which has been estimated from only a small number of measurements, $\psi$ is street orientation angle. 
The multiple screen diffraction loss was estimated by Walfisch and Bertoni for the case when the base station antenna is above the rooftops i.e. $h_{b}>h$. This has also been extended by COST to the case when the antenna is below rooftop height, using an empirical function based on measurements.

\section{Results \& Discussion}

The observed signal levels have been converted into path losses and compared with path losses predicted from Hata, Lee and COST 231 Walfisch \& Ikegami methods. Some typical figures of path loss comparison with the above prediction methods along with variation of path loss exponents with distance are shown in figures 5-10 for NN-1 and UA and PVR base stations. Figures 5,7 and 9 show the comparison of observed path loss with the above prediction methods for NN-1, UA and PVR base stations and figures 6 , 8 and 10 show the corresponding variation of path loss exponents. In figure 5 observed path loss at distances close to the transmitter varies from 80 to $130 \mathrm{~dB}$ and beyond $1 \mathrm{~km}$ is confined between 120 and $140 \mathrm{~dB}$. In figure 7 for UA base station at closer distances the variation of path loss is 80 to $120 \mathrm{~dB}$ and at larger distances the variation is 110 to 140 dB. Here the path loss is plotted up to distances of $2.5 \mathrm{~km}$. In figure 9 the path loss at distances below $500 \mathrm{~m}$ varies substantially and at later distances it is confined between 110 to $145 \mathrm{~dB}$. Figure 6 shows the variation of path loss exponent with distance for NN-1 base station. At distances very close to the transmitter exponent of 8 is seen and falls steeply to a value of 2.5 at a distance of $200 \mathrm{~m}$ and then varies between 3.0 and 4.0 for the remaining distances of $1.6 \mathrm{~km}$. In figure 8 also the exponent also falls from 8 to 2.5 at a distance of $400 \mathrm{~m}$ and then gets confined between 2.5 and 4.0 for the remaining distances. In figure 10 also the path loss exponent falls steeply from 8 to 3.5 around $300 \mathrm{~m}$ and thereafter remains stable for the remaining distances. 
The path loss exponent shown in figures 6 and 8 has been deduced based on the following methodology. Path loss exponents from the data have been deduced from the equation 1 shown below using the observed path losses for various distances and $\mathrm{L}_{0}$ depicting the path loss at one meter distance.

$$
\mathrm{L}=\mathrm{L}_{0}+10 \mathrm{n} \log \mathrm{d}+\mathrm{S}
$$

Where $\mathrm{L}$ is the path loss deduced for various distances, $\mathrm{L}_{0}$ is the path loss at one meter and $\mathrm{d}$ is the distance in meters; $\mathrm{S}$ is the shadow fading in $\mathrm{dB}$. Using the approach given in reference [15] of Erceg et al., the path loss exponent $n$ has been deduced. In the above equation $\mathrm{L}_{0}=20 \log \left(4 \prod \mathrm{d}_{0} / \lambda\right)$ where $\lambda$ is the wavelength corresponding to $900 \mathrm{MHz}$ and $\mathrm{d}_{0}$ is taken as one meter. $\mathrm{S}$ is the shadow fading variation and varies from location to another within given macrocell. It tends to be Gaussian in a given macro cell denoting shadow fading as lognormal. It can be expressed as $\mathrm{s}=\mathrm{y} \sigma$, where $\mathrm{y}$ is a zero mean Gaussian variable of unit standard deviation and $\sigma$ the standard deviation of $\mathrm{s}$ is itself a Gaussian variable over macrocells. $\mathrm{L}$ is taken from the observed path loss values. Using this methodology the path loss exponent 'n' has been deduced.

\subsection{Discussion on statistical analysis parameters for all base stations}

Instead of comparing individually the results of each base station with the predicted values statistical parameters like root mean square error(RMSE), coefficient of

determination $\left(\mathrm{R}^{2}\right)[16]$, average hit rate error(AHRE) of prediction errors have been 
deduced. The errors between measured data and predicted values for each location point have been deduced and converted into RMSE, $\mathrm{R}^{2}$ and AHRE.

The RMSE function of this prediction error is as follows

$\mathrm{E}\left(\mathrm{L}_{0}, \gamma\right)=\sqrt{\frac{1}{N} \sum_{i=1}^{N}\left[e(d i]^{2}\right.}$

Where, $\mathrm{N}$ is number of measured points, $\mathrm{e}\left(\mathrm{d}_{\mathrm{i}}\right)$ is the prediction error. $\mathrm{L}_{0}, \gamma$ are Lee model parameters. $\mathrm{L}_{0}$ is a median path loss at $1 \mathrm{~km}$ distance; $\gamma$ is the slope of the path loss curve. The second parameter i.e. coefficient of determination $\left(\mathrm{R}^{2}\right)$ is defined as the square of the correlation between measured and predicted path losses. It is defined as

$$
\mathrm{R}^{2}=1-\frac{\sum_{i=1}^{N}\left[e\left(d_{i}\right)\right]^{2}}{\sum_{i=1}^{N}[P L(d i)-\overline{P L}]^{2}}
$$

Where $\overline{P L}$ is the mean path loss of the measured signal, PL $\left(\mathrm{d}_{\mathrm{i}}\right)$ is the path loss observed at various distances

\subsection{Total Hit rate (THR) and Average Hit Rate Error (AHRE)}

Hit rate metrics were introduced by Owadally et al [17] to complement the first order metrics for evaluating the prediction models. They have used location specific total hit rate to evaluate the quality of prediction model. It is defined as the percentage of locations at which the predictions and measurements agree for a particular path loss threshold i.e. locations having state $(1,1)$ or $(0,0)$. It is defined as

$\operatorname{THR}\left(\mathbf{L}_{\mathbf{T}}\right)=\left[\frac{\sum_{i} U\left(m_{i}\right) \cdot U\left(p_{i}\right)}{N_{T}}+\frac{\sum_{i} \bar{U}\left(m_{i}\right) \cdot \bar{U}\left(p_{i}\right)}{N_{T}}\right]$ 
Where $\mathrm{U}\left(\mathrm{m}_{\mathrm{i}}\right)$ and $\mathrm{U}\left(\mathrm{p}_{\mathrm{i}}\right)$ denote step functions corresponding to measured and predicted values, $\mathrm{N}_{\mathrm{T}}$ refers to the total number of points compared. The method is useful in assessing the validity of a model where coverage is determined simply by a threshold value of path loss. Ostlin et al [18] extended the concept by introducing the parameter average total hit rate error (AHRE) to compare different models. It is mean deviation from hundred percent THR and is expressed as

$$
A H R E=\frac{1}{N_{L T}} \sum_{L_{T, \min }}^{L_{T \max }} 100 \%-\operatorname{THR}\left(L_{T}\right)
$$

Where $\mathrm{L}_{T}$ is the path loss threshold and $\mathrm{N}_{\mathrm{LT}}$ is the number of THR points. $\mathrm{L}_{T \min }$ and $\mathrm{L}_{T \max }$ are taken from the observed path loss values and are between 80 and $150 \mathrm{~dB}$. A model is said to perform well when it exhibits a lower value of AHRE. Ostlin et al [11] have interpreted AHRE as the area between the THR curve and $100 \%$.

Initially RMSE and $\mathrm{R}^{2}$ for all the above three models have been deduced for all the base stations utilized in this study. They are given in table 2. A perusal of the table showed that for Hata method in urban zones the RMSE values varied from 8.5 to 17.58 , for Lee method they varied from 8.5 to 16.46 , for COST $231 \mathrm{WI}$ method the variation was between 8.42 to 14.68 . Similarly $\mathrm{R}^{2}$ varied from 0.2 to 0.7 for Hata method, 0.01 to 0.48 for Lee method, and 0.17 to 0.49 for COST 231 WI method. In the suburban region Hata's method exhibited RMSE of 8.12 to 15.16 , Lee 9.39 to 16.88 and COST $231 \mathrm{WI}$ method 9.72 to 13.95 . All the three methods exhibited more or less similar values on the lower side of RMSE but on the higher side the values went up to $17 \mathrm{~dB}$. Even $\mathrm{R}^{2}$ which indicates the goodness of fit was not very high and turned out to be negative for TXT base station in suburban zone for all the three methods, and for CWI and Lee methods for GRN base station. Since both the statistical parameters were not presenting a clear 
picture of agreement we have attempted to tune the prediction methods. The selection of the Lee model for tuning is based on simplicity, ease of tuning, applicability to the prevailing conditions

under test, prediction accuracy and parameters involved. Since Lee model has been recognized by the wireless industry as one of the most accurate propagation model in the $900 \mathrm{MHz}$ band it has been chosen for tuning. The following section gives brief outline of methodology of tuning.

\subsection{Methodology of tuning the Lee model}

The Lee area to area model is given by

$$
\mathrm{L}(\mathrm{dB})=\mathrm{L}_{0}+\gamma \cdot \log (\mathrm{d})-10 \log \left(\mathrm{F}_{0}\right)
$$

where $\mathrm{L}$ represents the path loss value at a given distance, $\mathrm{L}_{0}$ is a median path loss at 1 $\mathrm{km}$ distance, $\gamma$ is the slope of the path loss curve and $\mathrm{F}_{0}$ is the adjustment factor comprised of several factors, $F_{0}=F_{1} F_{2} F_{3} F_{4} F_{5}$ which have been explained in the original Lee papers. The first two terms represent the impact from man made structure and the third term represents terrain related parameter.

The importance of tuning comes in the fact that every region will have its own characteristic slope and intercept values due to the different man made constructions. The layout of buildings and the street widths in this part of our country especially Delhi are some what peculiar in some of these zones the buildings are not uniformly distributed with variable heights. This could be the main reason why the above prediction methods deviated largely. The parameters of Lee method were developed over western countries where construction of buildings and material used are very much different from that over Delhi region where measurements were made. Hence tuning of Lee model is necessary and important in the present context. Here an attempt is made to modify the $\mathrm{L}_{0}$ and $\gamma$ of Lee method based on these observed values. For tuning purposes the main criterion is to minimize RMSE function. It should be differentiated partially with respect to their 
coefficients that achieve this minimization. To obtain Lee models parameters $\left(\mathrm{L}_{0}, \gamma\right)$ that optimize equation (1), $\mathrm{N}$ equations based on errors corresponding to $\mathrm{N}$ measured points should be solved.

The above $\mathrm{N}$ equations are solved using a Mat lab program to get $\mathrm{L}_{0}$, and $\gamma$.

\subsection{Discussion of Tuned results}

The values of Tuned Lee path loss model parameters $\mathrm{L}_{0}, \gamma$ were computed and tabulated in table 2. It shows that $\mathrm{L}_{0,} \gamma$ values for urban areas are high compared with suburban areas. In the present study tuned Lee parameter $\mathrm{L}_{0}$ varied from 107 to 117 in urban regions and 98 to 105 in suburban regions. The parameter $\gamma$ varied from 31 to 43 over urban regions and 27 to 31 over suburban regions. The average value of $\gamma$ over urban region is 36.78 and over suburban region are 29.0. The original value of $L_{0}$ proposed by Lee varied from 104 in Newark, 110 in Philadelphia and 124 in Tokyo and in suburban regions it is 101.7. The parameter $\gamma$ varied from 36.8 to 30.5 in urban areas it is 38.5 . Paiboon et al. in Bangkok city [19] reported $\mathrm{L}_{0}$ of $111 \mathrm{~dB}$ and $\gamma$ of 36 . The corresponding values reported over Taipei are [20] are $121 \mathrm{~dB}$ and 38, over Dhaka [21] are 126 and 30. Compared with the values reported in literature $\mathrm{L}_{0}$ values in the present study match with those reported in the literature whereas the $\gamma$ values are slightly higher than those reported in literature.

The main difference between tuned values in the present study and original Lee parameters is with reference to the parameter $\gamma$. We feel present set of $\gamma$ values are more representative of urban regions prevailing over Delhi. Out of the two parameters $\gamma$ is more important as it represents the rate of fall of signal level/path loss and these values can be used by cellular operators in models while using radio planning tools over this 
region and similar regions. Ali [22] over the urban desert regions of Saudi Arabia made an attempt to tune the values of Lee method on the basis of Tetra measurements in the $400 \mathrm{MHz}$ band. They observed values of $\gamma$ ranging from 23.47 to 33.8 . These values probably could be due to the different nature of urban environment and frequency used in this study is $400 \mathrm{MHz}$. The $\mathrm{L}_{0}$ values reported are 2 to 48 . The value 2 appears to be on the lower side. In this study the standard deviation (RMSE) varied from 3 to 4 in urban and suburban areas with average RMSE of 3.961 and $\mathrm{R}^{2}$ varied from 0.54 to 0.78 . In the present study after tuning RMSE varied from 5 to 9 and $\mathrm{R}^{2}$ showed variations from 0.29 to 0.79 in urban regions. In suburban regions the corresponding variations are $5.8-10$ for RMSE and 0.29-0.39 for $\mathrm{R}^{2}$. The tuned values of RMSE and $\mathrm{R}^{2}$ are shown in table 1 . The average RMSE for tuned Lee method in urban and suburban zones is found to be 7.69 and 7.68 respectively.

From the table 2, comparison of tuned values of Lee method with other models showed that the RMSE and $\mathrm{R}^{2}$ values of tuned Lee model performed better than the traditional models like Hata and COST 231 WI methods. The averaged RMSE value for all the base stations for the tuned Lee model is $7.69 \mathrm{~dB}$ and average RMSE value for Hata and COST 231 WI models are 11.03, 11.39 respectively for urban regions. Overall tuned Lee model performed well. In the case of COST 231 WI model the parameters used are, separation between buildings $b=10 \mathrm{~m}$, average height of the buildings $\mathrm{hr}=6 \mathrm{~m}$. The average RMSE value of tuned Lee model is less compared to Hata and COST 231 WI. The $\mathrm{R}^{2}$ value of tuned Lee model is better compared to Hata and COST 231 WI models. In suburban environments COST $231 \mathrm{WI}$ model over estimated the path loss 
values. Hence RMSE and $\mathrm{R}^{2}$ values are not given for TXT tower MPR and GRN base stations.

Table 3 denotes the AHRE values for all the base stations for the three prediction methods. In the case of urban region for Hata's method AHRE varied from 8 to 20.8, 8.16to 19.31 for CWI method, 5 to 15.69 for tuned Lee method. For PVR base station all the three methods exhibited lowest values ranging from 5.7 for tuned Lee, 8.16 for CWI and 11.13 for Hata's method. Highest values were observed for NN 2 base station. For suburban regions, AHRE varied from 12 to 21.6 for Hata's method, 15 to 21.2 for CWI method, 9 to 10 for tuned Lee method. Urban zones exhibited better values than suburban values. The highlight of the study is the lowest values (improved model performance) exhibited by tuned Lee method compared with Hata and COST 231 WI for all the base stations. The average AHRE in urban region for all the base stations are Hata $=14.16$, COST 231 WI method $=12.7$, tuned Lee method $=10.26$. In the case of suburban region the corresponding values are $16.27,17.6 \& 10.4$. These tuned value of Lee method can also be applied to the similar type of medium urban environment prevailing over various parts of India and other countries.

\section{Conclusions}

Signal level measurements were conducted utilizing 7 urban and 4 suburban GSM base stations in the national capital region of Delhi in the $900 \mathrm{MHz}$ band and the observed path losses were compared with prediction models like Hata, COST 231 WI and Lee methods. The comparison of predictions with observed values have been given in terms of RMSE, $\mathrm{R}^{2}$ and AHRE. Based on the observed path losses the parameters of Lee method were tuned and new values of parameters $L_{0}$ and $\gamma$ were deduced for these 
regions. The values of $\mathrm{L}_{0}$ deduced vary from 104 to 117 in urban regions and 98 to 114 in suburban regions. The parameter $\gamma$ varied from 14 to 26 over urban regions and 13 to 16 over suburban regions. The statistical metrics of tuned Lee method were seen to outperform the Hata and COST 231 WI methods significantly.

\section{References:}

1. L.C. Fernandes , A.J. Martins Soares, " Simplified characterization of the urban propagation environment for path loss calculation, IEEE Antennas and wireless propagation letters, 9,pp 24-27, 2010

2. Ericsson radio systems AB,TEM STM cellplanner 3.4,User guide, 2001

3. Digital mobile radio towards future generation systems, COST 231 group final report, COST telecom secretariat, COST@postman.dg13.cec.be

4.R.D. Vieira, R.C.D.Paiva , J. Hulkkonen, R. Jarvela, R.F. Iida, M.Saily, F.M.L.Tavares, $K$. Niemela "GSM evolution importance in re-farming $900 \mathrm{MHz}$ band" in $72^{\text {nd }}$ IEEE international conference on vehicular technology (VTC) 6-9 $9^{\text {th }}$ sept, 2010 fall, pp 1-5., DOI:10.1109/VETECF.2010.5594534.

5. D.J.Y. Lee and W.C.Y. Lee, "Enhanced Lee model from rough terrain sampling data aspect" in $72^{\text {nd }}$ IEEE international conf on Vehicular technology (VTC), 6-9 $9^{\text {th }}$ Sep, 2010 fall, pp 1-5, DOI:10.1109/VETECF.2010.5594119

6. M. Hata "Empirical formula for propagation loss in land mobile radio services IEEE Transactions Vehicular Technology, vol.29, pp 317-325, 1980

7. John S.Seybold, “Introduction to RF Propagation” John Wiley \& sons, New Jersey pp 153-154,2005

8. W.C.Y. Lee "Mobile communication engineering Theory and applications", $2^{\text {nd }}$ ed., McGraw Hill, New York, pp 59-67, 1993

9. COST 231, Final report, Digital Mobile Radio: COST 231 View on the evolution Towards 3rd Generation systems, Commission of the European Communities and COST Telecommunications, Brussels, 1999

10. W C Y Lee, "Estimation of local average power of a mobile radio signal", IEEE Trans. Veh. Technology, Vol.VT-34, No.1, 1985

11.W C Y Lee, "Mobile communication design fundamentals", Howard W. Sams and Co., 1986

12. International Telecommunication union, "Field strength measurements along a route with geographical coordinate registrations, ITU-R recommendation SM.1708, April 2005

13. European Conference of Postal and Telecommunications Administrations, "ERC

Report,77, Field strength measurements along a route:, ERC REc.08(00), January 2000

14. International Telecommunication Union, "Generalized method for monitoring broadcasting signals and for the analysis of field strength variation. Application to 
ground wave propagation in MF band" ITU documents 3J/26-E and 3K/22-E, May2008

15. V. Erceg, S.Y. Tjandra, S.R. Parkoff, Ajay Gupta, Boris Kulic, A.A. Julius and Renee Bianchi, "An empirically based path loss model for wireless channels in suburbanenvironments", IEEE J Selected areas in Communications, vol.17, no.7, pp 1205-1211, 1999

16. R.E. Walpole and R.H. Myers, "Probability and statistics for Engineers and scientists, $3^{\text {rd }}$ edition, Macmillan, New York, pp 353, 1985

17. A.S. Owadallly, E. Montiel and S.R. Saunders S. R. "A comparison of the accuracy of propagation models using hit rate analysis", in $54^{\text {th }}$ IEEE conf on Vehicular Technology (VTC), vol.4, pp 1979-1983, 2001 fall

18. E. Ostlin, H.Suzuki and H.J. Zepernick, "Evaluation of the propagation model recommendation ITU-R P.1546 for mobile services in rural Australia IEEE Trans Vehicular Technology, vol.57,no.1, pp 38-51,2008

19. S. Phaiboon, P. Phokharatkul and S. Somkuarnpanit, Propagation-Path losses characterization for $800 \mathrm{MHz}$ cellular communications in Bangkok", in Proceeding of the IEEE Region 10 Conference(TENCON 99),vol. 2,pp 1209-1211,15-17september 1999 20. Chien-Chung Wang and Jhin-Fang Huang, Propagation path loss characterization for an $870 \mathrm{MHz}$ cellular CDMA system in Taipei city", in Proc. IEEE international symposium on Microwave, Antenna, Propagation and EMC Technologies for Wireless communications, pp 790-793, 16-17 Aug 2007

21. A.B.M. Siddique Hossain and Md. Ramjan Ali, "Propagation path losses characterization for $900 \mathrm{MHz}$ cellular communications in Dhaka city" in proc. of $9^{\text {th }}$ Asia-Pacific conference, vol.1, 21-24 Sept,2003.

22. Faihan D.Alotaibi and Ali A.A, “ Tetra Outdoor Large-Scale Received Signal Prediction Model in Riyadh City-Saudi Arabia" in proc. of IEEE Wireless and Microwave technology Conference (WAMICON) pp 1-5, 4-5 Dec,2006 
Table1. Base station specifications along with tuned Lee model parameters for urban regions of Delhi

\begin{tabular}{|c|c|c|c|c|c|}
\hline Urban region & $\mathrm{Hb}(\mathrm{m})$ & Lat & Long & $\begin{array}{l}\text { Tuned } \\
\text { Lee } \\
\mathrm{L}_{0}\end{array}$ & $\begin{array}{l}\text { Tuned Lee } \\
\gamma\end{array}$ \\
\hline $\begin{array}{l}\text { PVR(43.8dBm, } \\
2 \mathrm{dBi})\end{array}$ & 13 & 28.6738 & $\begin{array}{l}77.0888 \\
2\end{array}$ & 108.20 & 33.50 \\
\hline $\begin{array}{l}\text { UA }(43.8 \mathrm{dBm}, \\
2 \mathrm{dBi})\end{array}$ & 24 & 28.69655 & $\begin{array}{l}77.2136 \\
7\end{array}$ & 110.27 & 36.12 \\
\hline $\begin{array}{l}\text { NN1(43.8dBm, } \\
2 \mathrm{dBi})\end{array}$ & 14 & 28.66912 & $\begin{array}{l}77.3625 \\
6\end{array}$ & 117.70 & 38.16 \\
\hline $\begin{array}{l}\mathrm{NN} 2(43.8 \mathrm{dBm}, \\
2 \mathrm{dBi})\end{array}$ & 12 & 28.68908 & 77.3026 & 114.50 & 43.35 \\
\hline $\begin{array}{l}\text { SNT(43.8dBm, } \\
2 \mathrm{dBi})\end{array}$ & 18 & 28.58845 & $\begin{array}{l}77.1684 \\
8\end{array}$ & 115.40 & 38.46 \\
\hline $\begin{array}{l}\text { FBD(43.8dBm, } \\
2 \mathrm{dBi})\end{array}$ & 10 & 28.38071 & $\begin{array}{l}77.2972 \\
0\end{array}$ & 107.30 & 36.30 \\
\hline $\begin{array}{l}\mathrm{VKH}(43.8 \mathrm{dBm}, \\
2 \mathrm{dBi})\end{array}$ & 13 & 28.57132 & $\begin{array}{l}77.3280 \\
9\end{array}$ & 108.70 & 31.60 \\
\hline \multicolumn{6}{|l|}{$\begin{array}{l}\text { Suburban } \\
\text { region }\end{array}$} \\
\hline $\begin{array}{l}\text { TXT(43.8dBm, } \\
\text { 2dBi) }\end{array}$ & 43 & 28.47266 & $\begin{array}{l}77.5144 \\
7 \\
\end{array}$ & 98.54 & 28.20 \\
\hline $\begin{array}{l}\text { MPR(43.8dBm, } \\
2 \mathrm{dBi})\end{array}$ & 12 & 28.49434 & $\begin{array}{l}77.3245 \\
0\end{array}$ & 104.70 & 31.23 \\
\hline \multirow[t]{2}{*}{$\begin{array}{l}\text { GRN(43.8dBm, } \\
8 \mathrm{dBi})\end{array}$} & 20 & 28.4753 & $\begin{array}{l}77.0844 \\
5\end{array}$ & 105.20 & 27.82 \\
\hline & $\begin{array}{l}\mathrm{Hm}=1 . \\
5\end{array}$ & & & & \\
\hline
\end{tabular}


Table 2. Comparison of prediction methods used in the present study based on RMSE and $\mathrm{R}^{2}$

\begin{tabular}{|l|l|l|l|l|l|l|l|l|}
\hline $\begin{array}{l}\text { Urban } \\
\text { region }\end{array}$ & Hata & CWI & Lee & $\begin{array}{l}\text { Tuned } \\
\text { Lee }\end{array}$ & Hata & CWI & Lee & $\begin{array}{l}\text { Tune } \\
\mathrm{d} \\
\text { Lee }\end{array}$ \\
\hline PVR & 12.14 & 8.42 & 9.47 & 5.52. & 0.335 & 0.488 & 0.014 & 0.766 \\
\hline UA & 10.50 & 8.99 & 8.50 & 8.06 & 0.239 & 0.443 & 0.493 & 0.552 \\
\hline NN- 1 & 8.60 & 13.68 & 12.45 & 6.83 & 0.681 & 0.177 & 0.317 & 0.794 \\
\hline NN- 2 & 8.54 & 12.8 & 16.46 & 9.28 & 0.753 & 0.29 & -1.22 & 0.293 \\
\hline SNT & 8.46 & 8.79 & 10.2 & 6.62 & 0.493 & 0.494 & 0.318 & 0.691 \\
\hline FBD & 11.42 & 9.96 & 13.05 & 7.61 & 0.304 & 0.317 & -0.230 & 0.579 \\
\hline VKH & 17.58 & 14.68 & 10.50 & 9.96 & 0.433 & 0.241 & 0.488 & 0.540 \\
\hline $\begin{array}{l}\text { Suburban } \\
\text { region }\end{array}$ & & & & & & & & \\
\hline TXT & 15.16 & 13.95 & 15.5 & 6.47 & -0.746 & -0.776 & -0.11 & 0.395 \\
\hline MPR & 9.79 & 12.92 & 16.88 & 10.78 & 0.652 & 0.056 & -0.567 & 0.295 \\
\hline GRN & 8.12 & 9.72 & 9.39 & 5.80 & 0.256 & -0.747 & -0.682 & 0.355 \\
\hline
\end{tabular}

Table 3. Average hit rate error for all the base stations

\begin{tabular}{|l|l|l|l|}
\hline Urban region & Hata & CWI & Tuned Lee \\
\hline PVR & 11.13 & 8.16 & 5.70 \\
\hline UA & 12.68 & 10.33 & 10.32 \\
\hline NN- 1 & 8.06 & 12.11 & 6.84 \\
\hline NN- 2 & 15.44 & 13.53 & 12.908 \\
\hline SNT & 17.06 & 19.31 & 15.69 \\
\hline FBD & 13.97 & 9.50 & 7.48 \\
\hline VKH & 20.8 & 16.34 & 12.90 \\
\hline Average & 14.16 & 12.70 & 10.26 \\
\hline $\begin{array}{l}\text { Suburban } \\
\text { region }\end{array}$ & & & \\
\hline TXT & 21.6 & 21.12 & 10.03 \\
\hline MPR & 12.9 & 15.57 & 12.19 \\
\hline GRN & 14.33 & 16.31 & 9.11 \\
\hline Average & 16.27 & 17.60 & 10.4 \\
\hline
\end{tabular}


Fig 1. Google map depicting all base stations

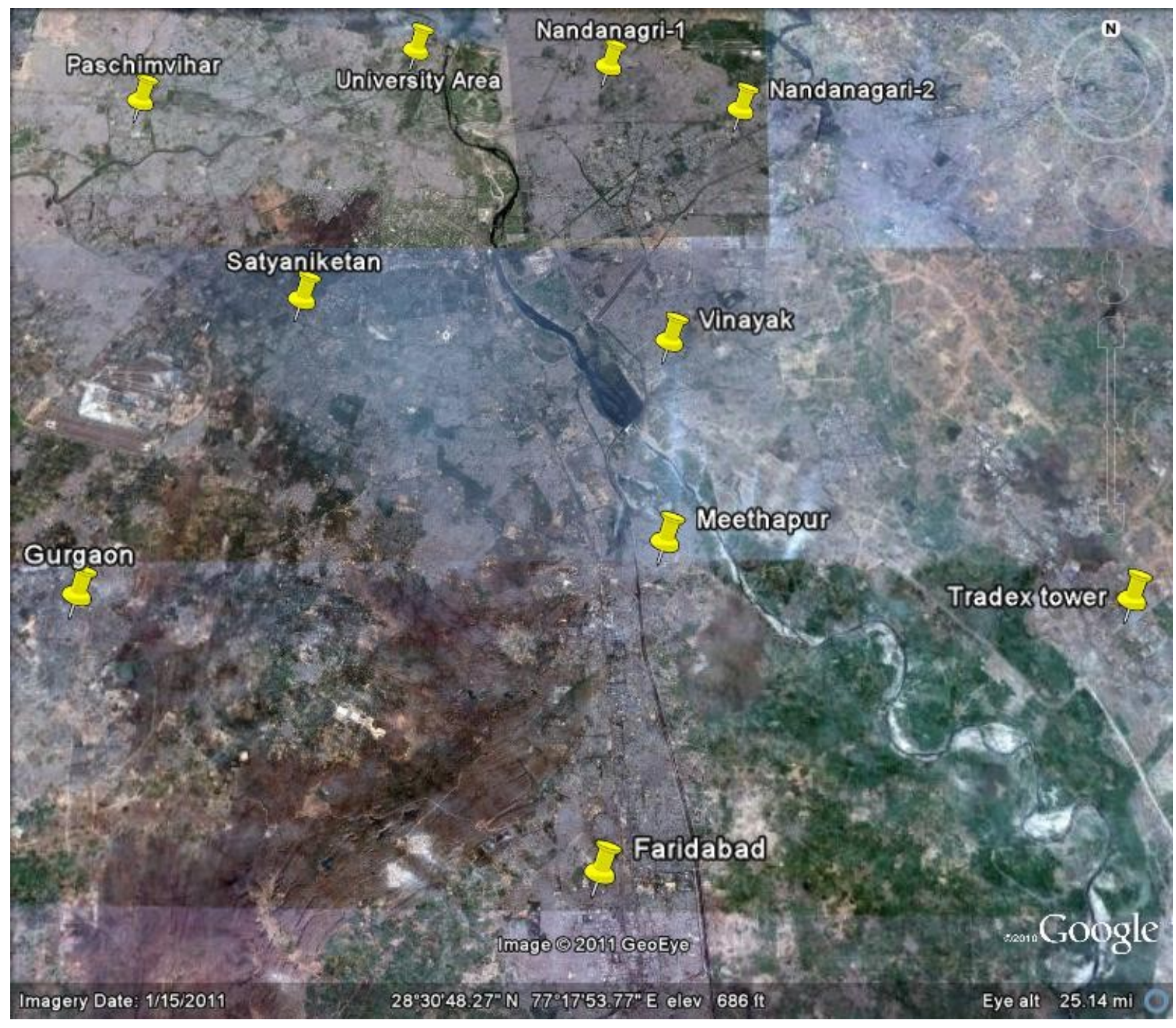


Fig 2.(a) Measured route for NN-1 base station

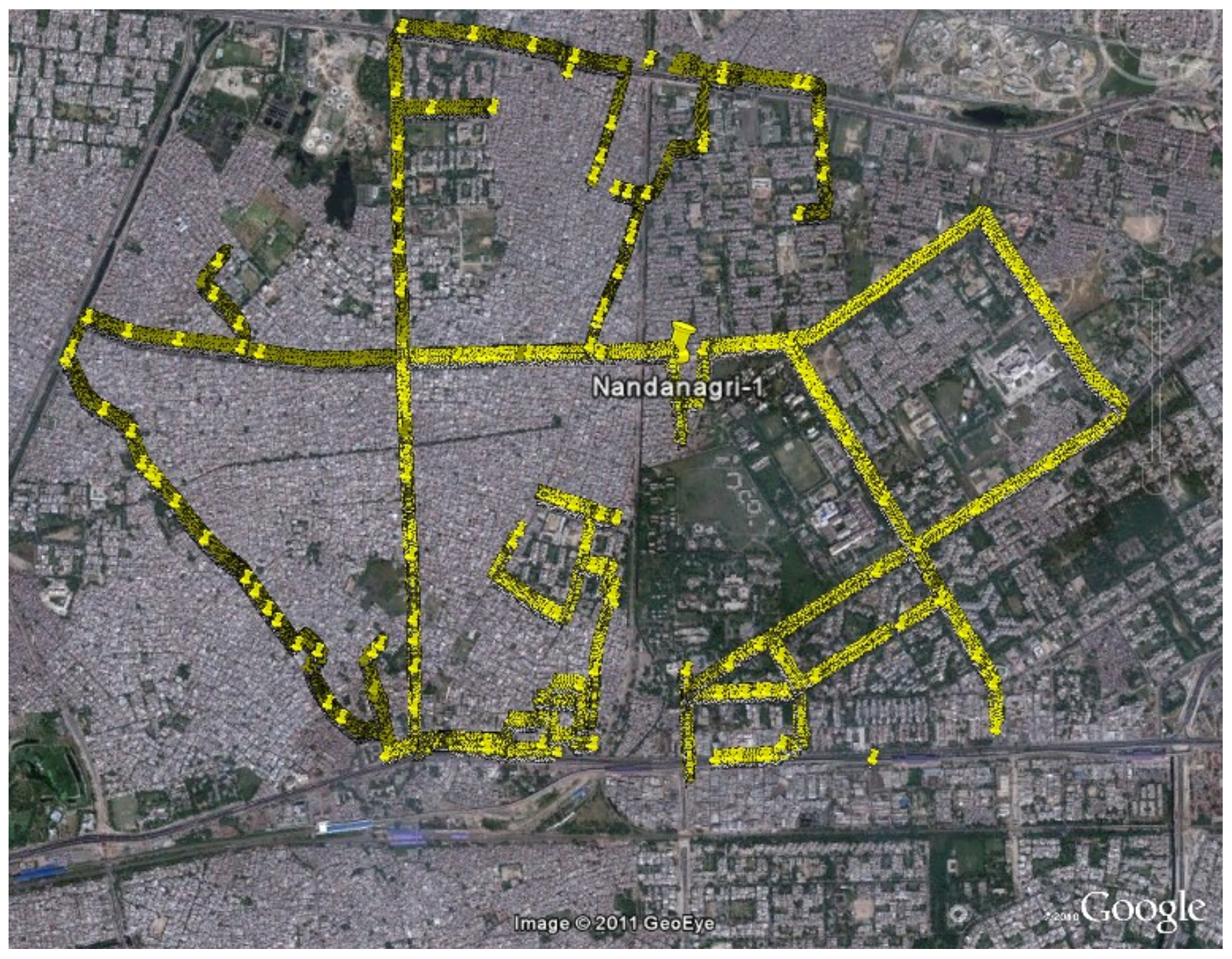


Fig 2(b) Measured route for Paschim vihar base station

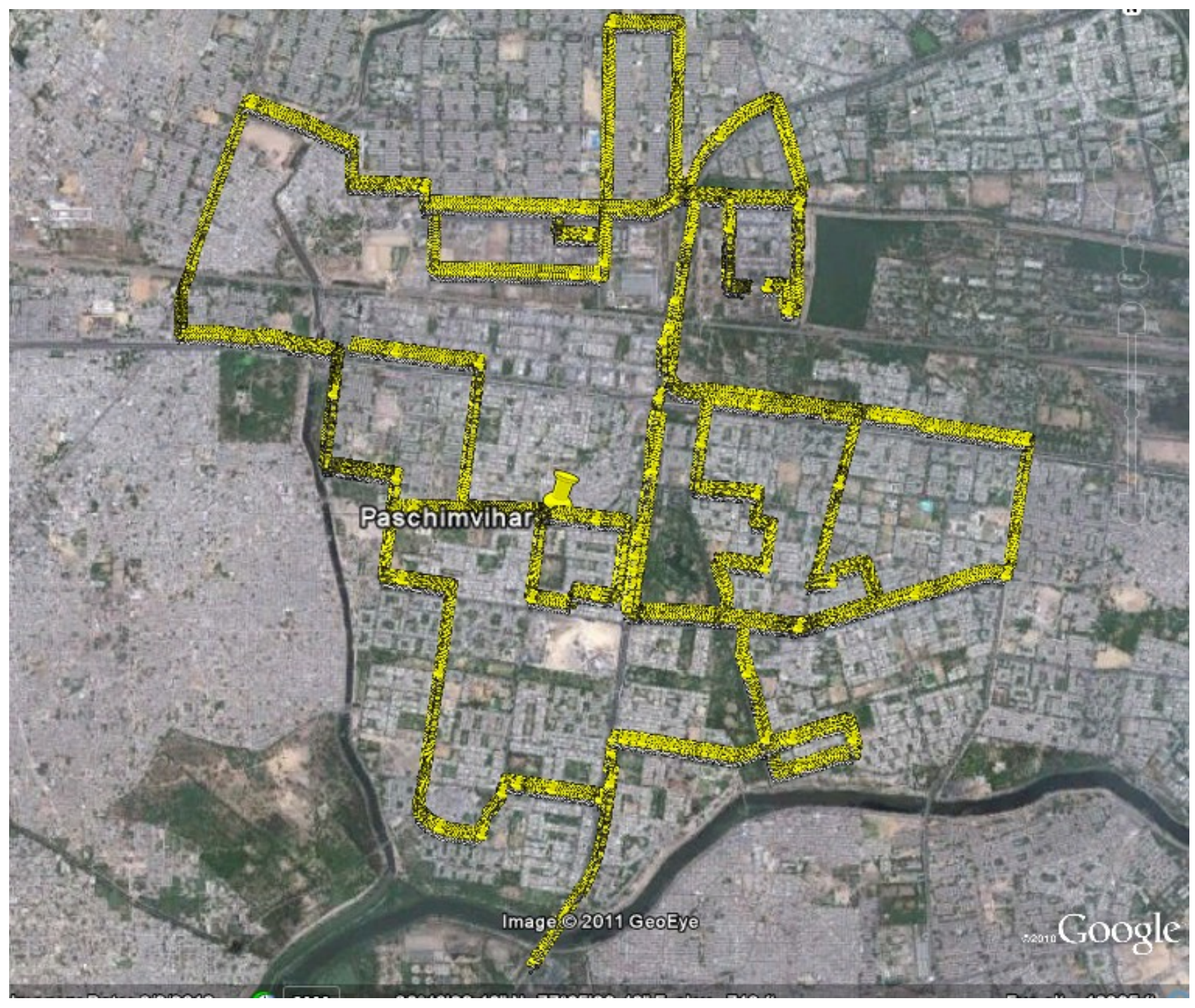


Fig 2(c) Measured route for Tradex tower base station

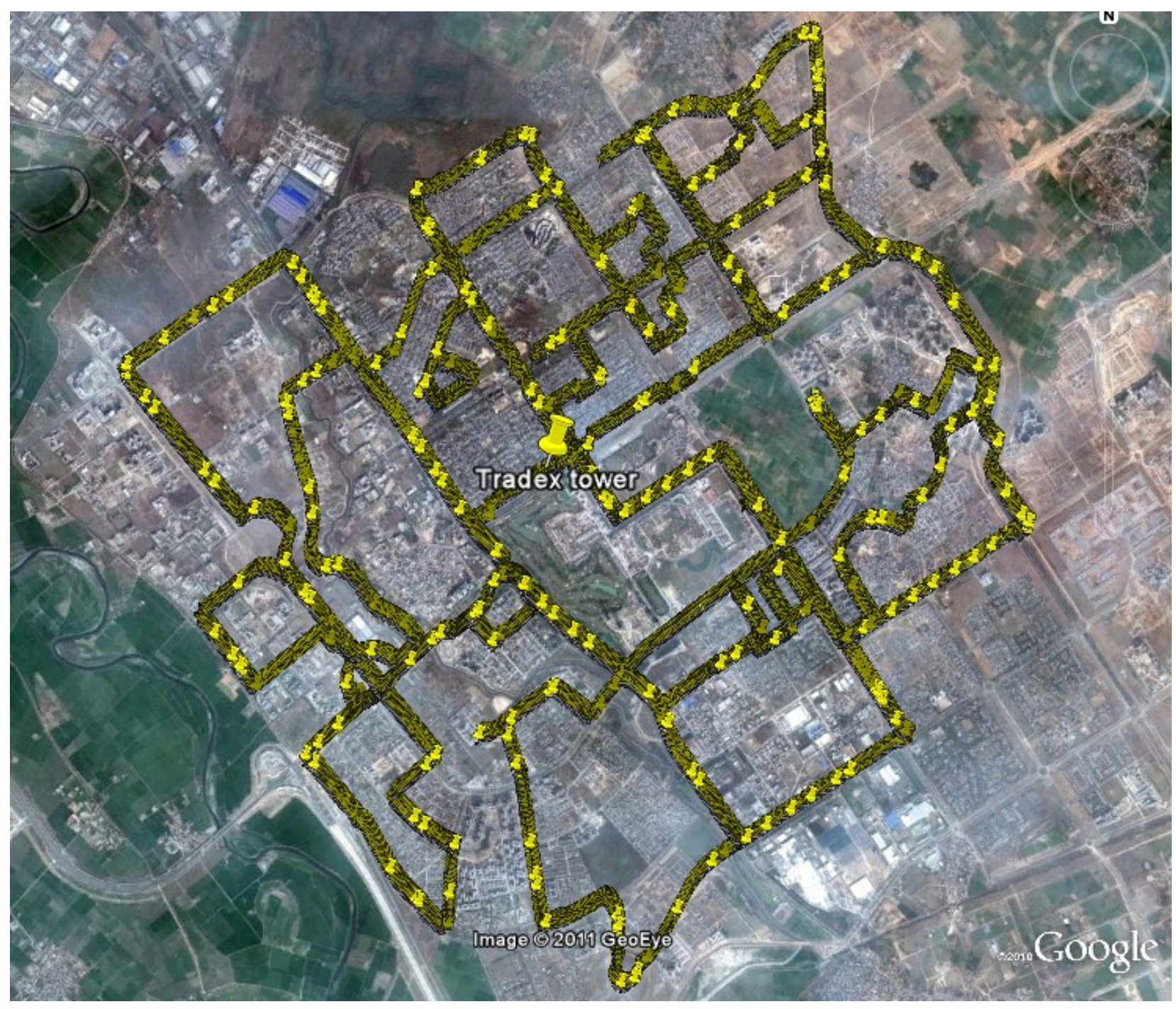


Fig 3. Clutter features around NN-1 base station

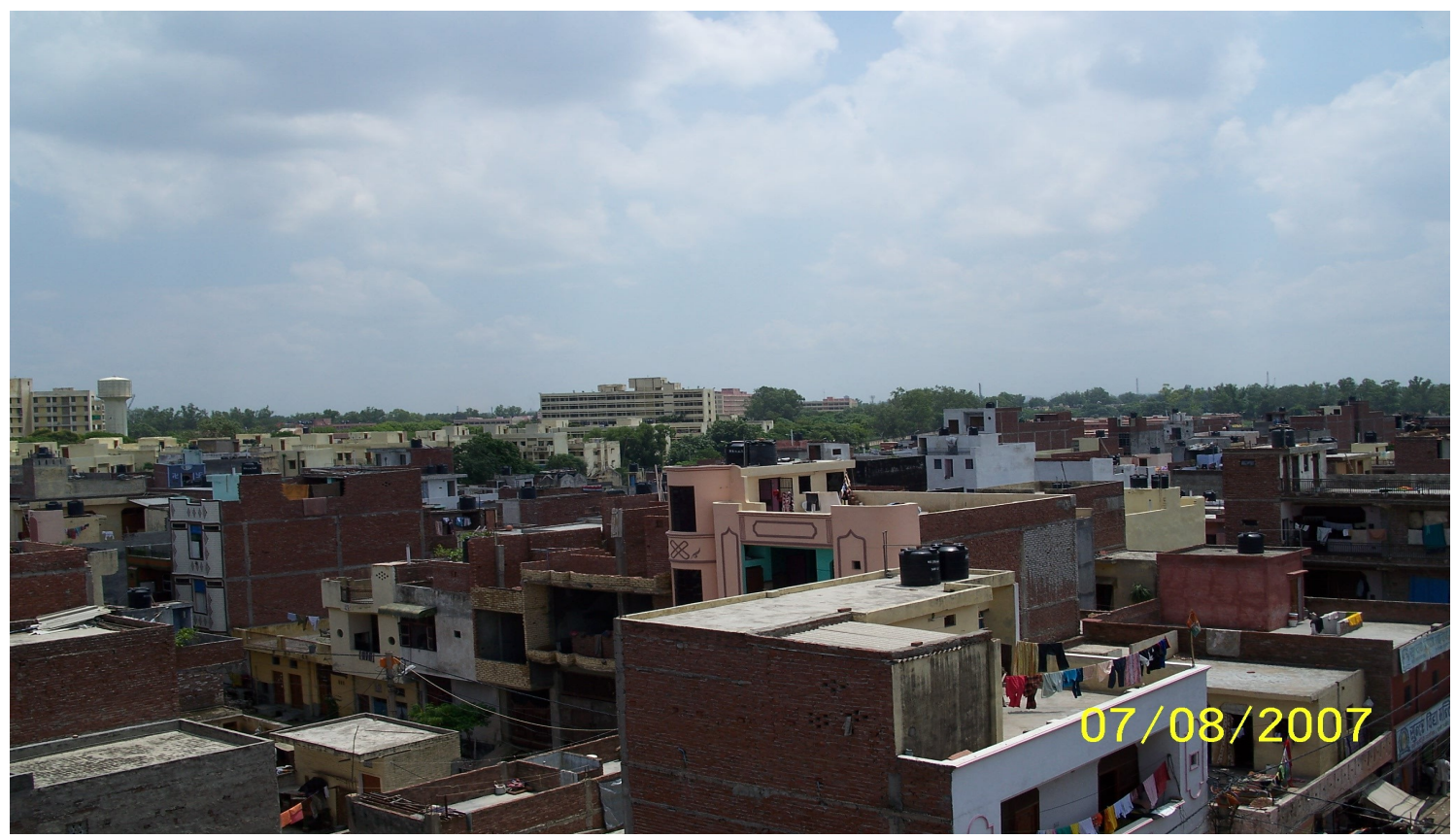

Fig4. Clutter features around UA base station

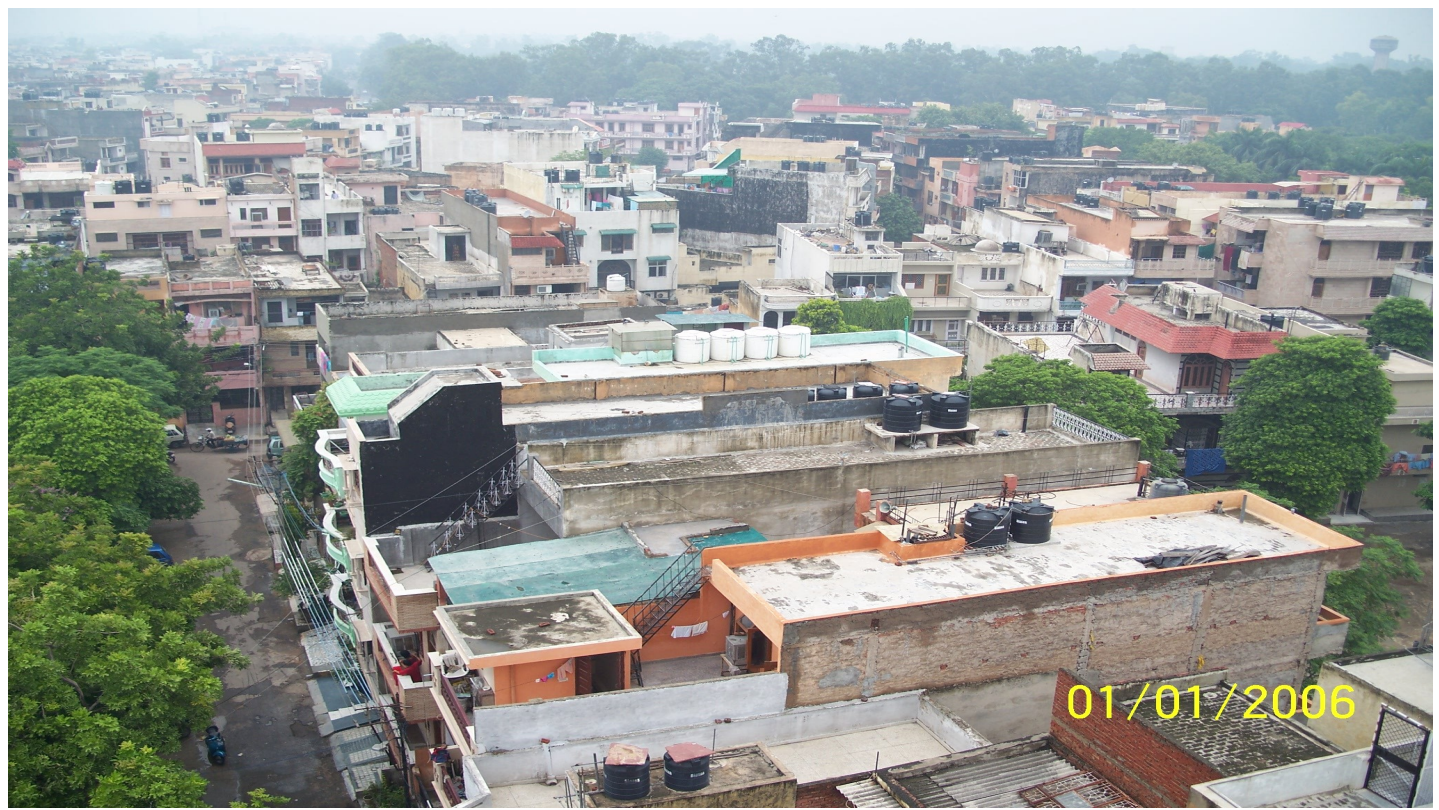


Fig 5. Comparison of observed path losses with those deduced from various prediction methods for $\mathrm{NN}-1$ base station

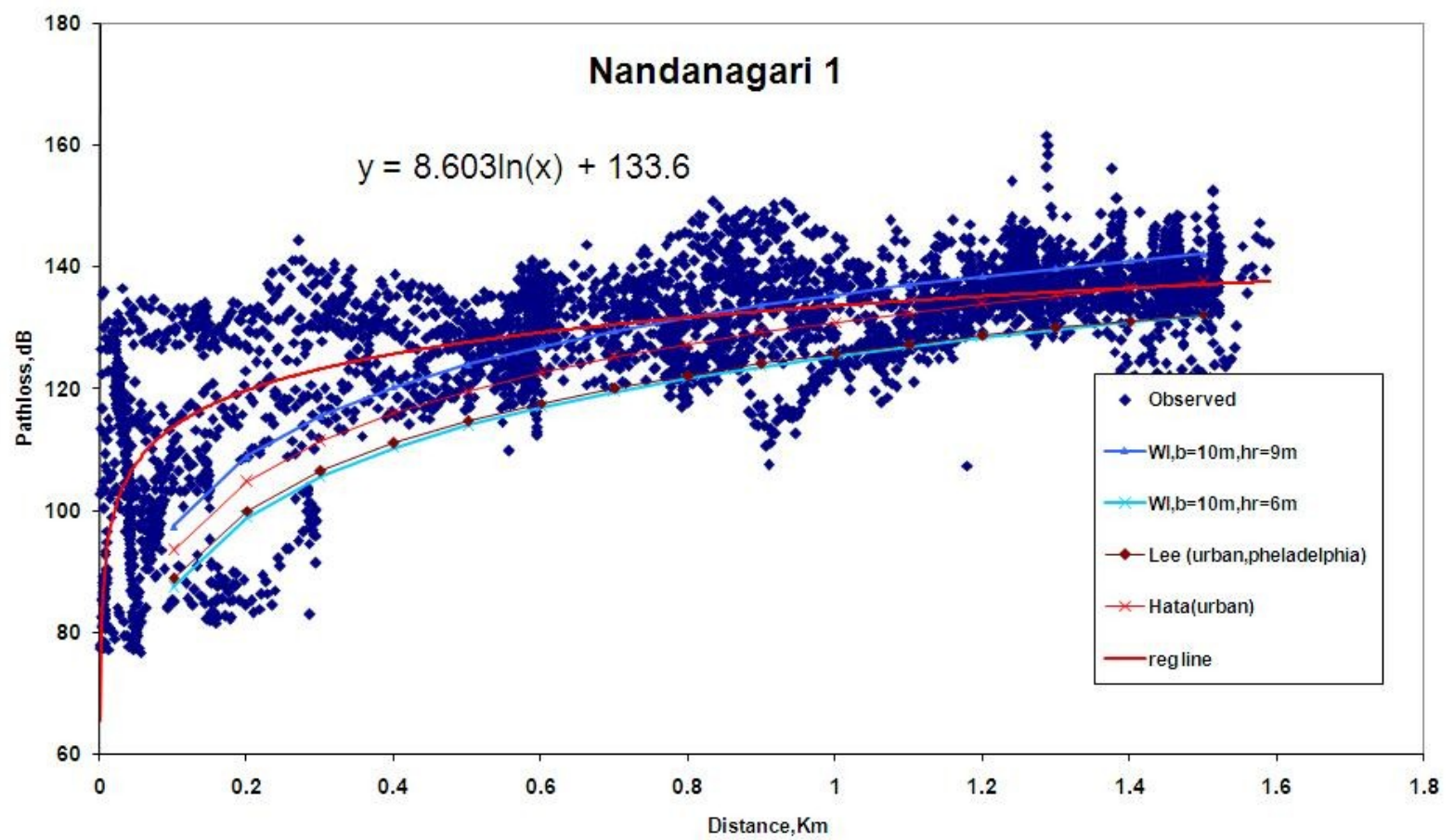

Fig 6. Variation of path loss exponent as a function of distance for NN-1 base station

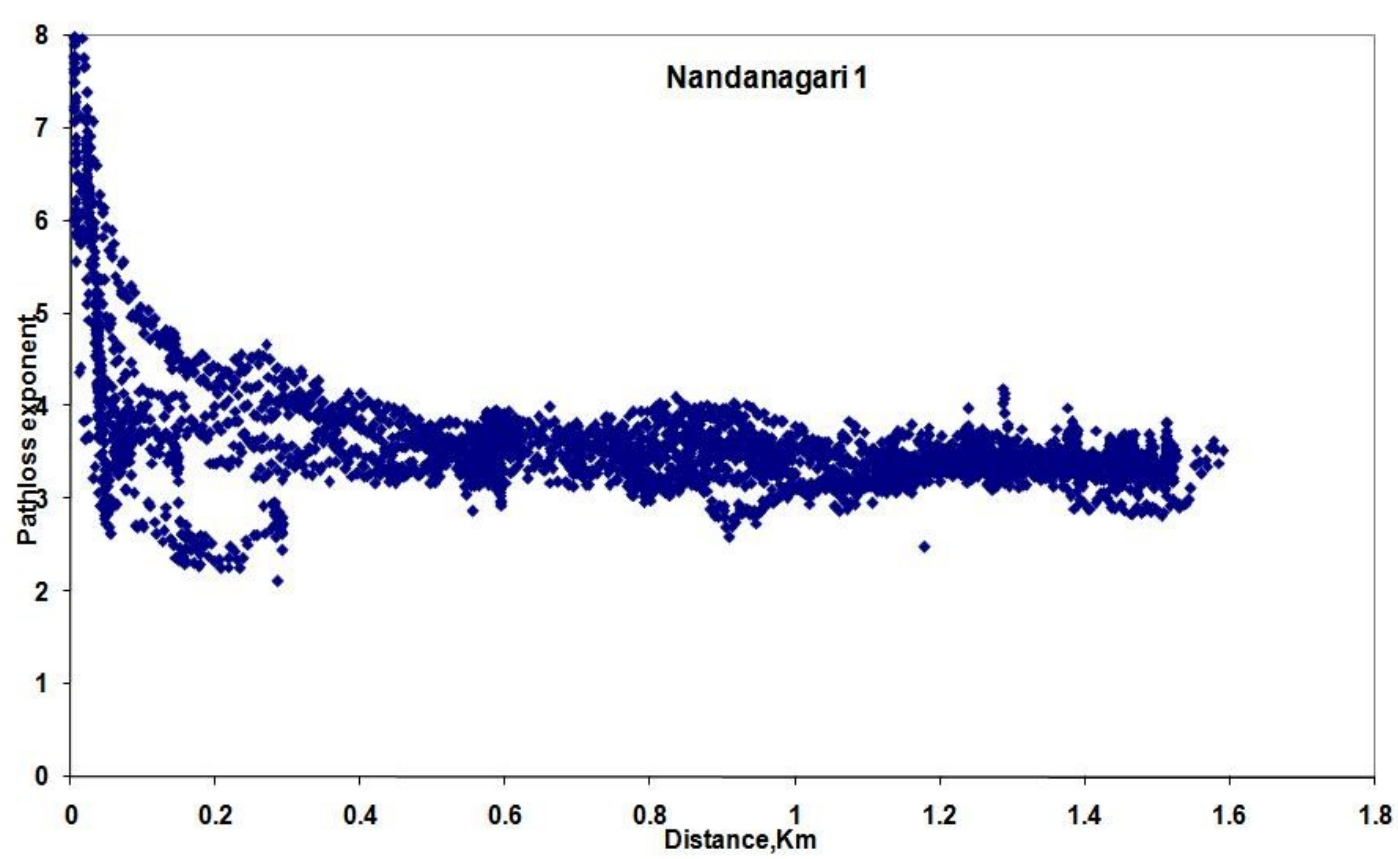


Fig 7. Comparison of observed path losses with those deduced from various prediction methods for UA base station

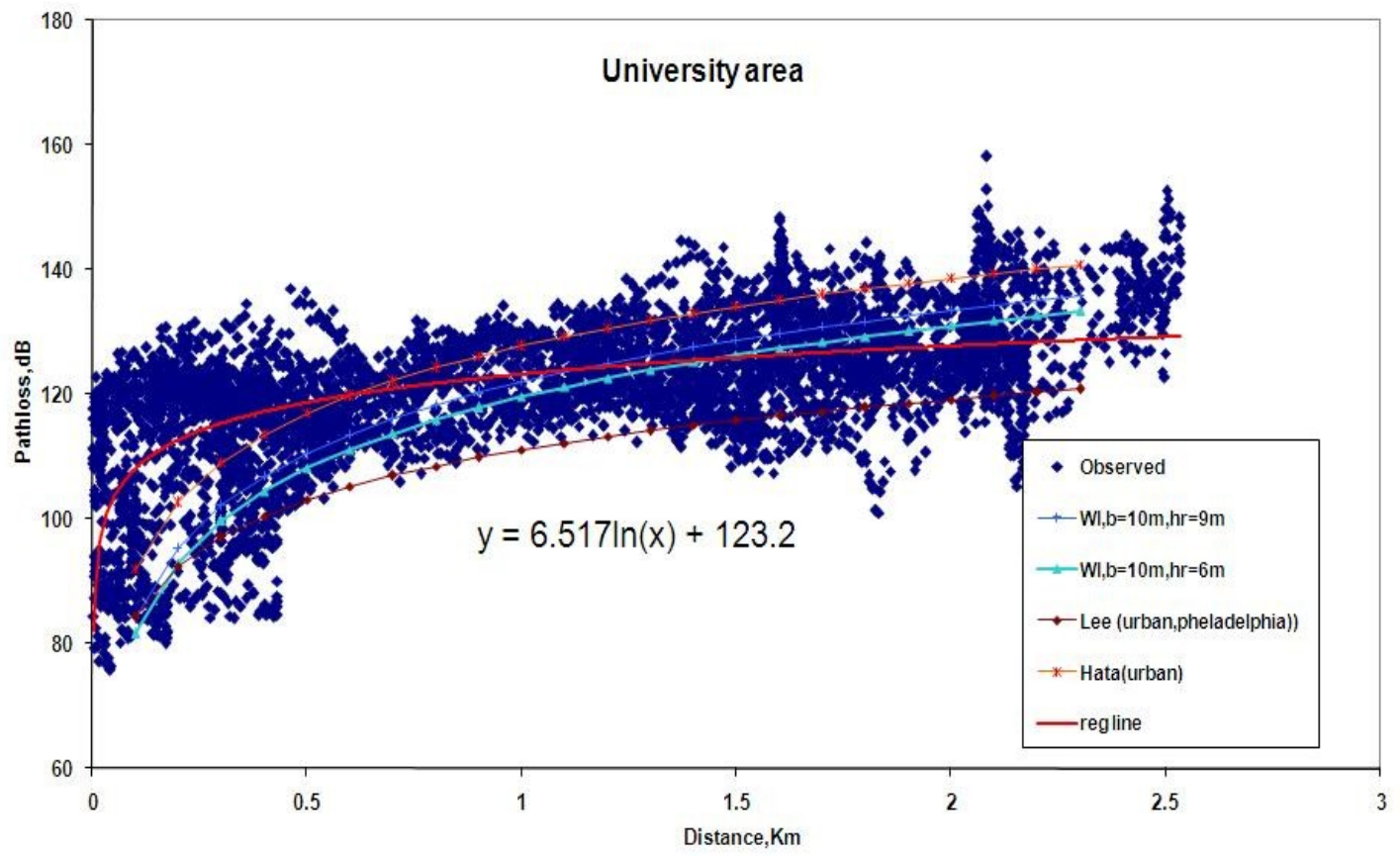


Fig 8. Variation of path loss exponent as a function of distance for NN-1 base station

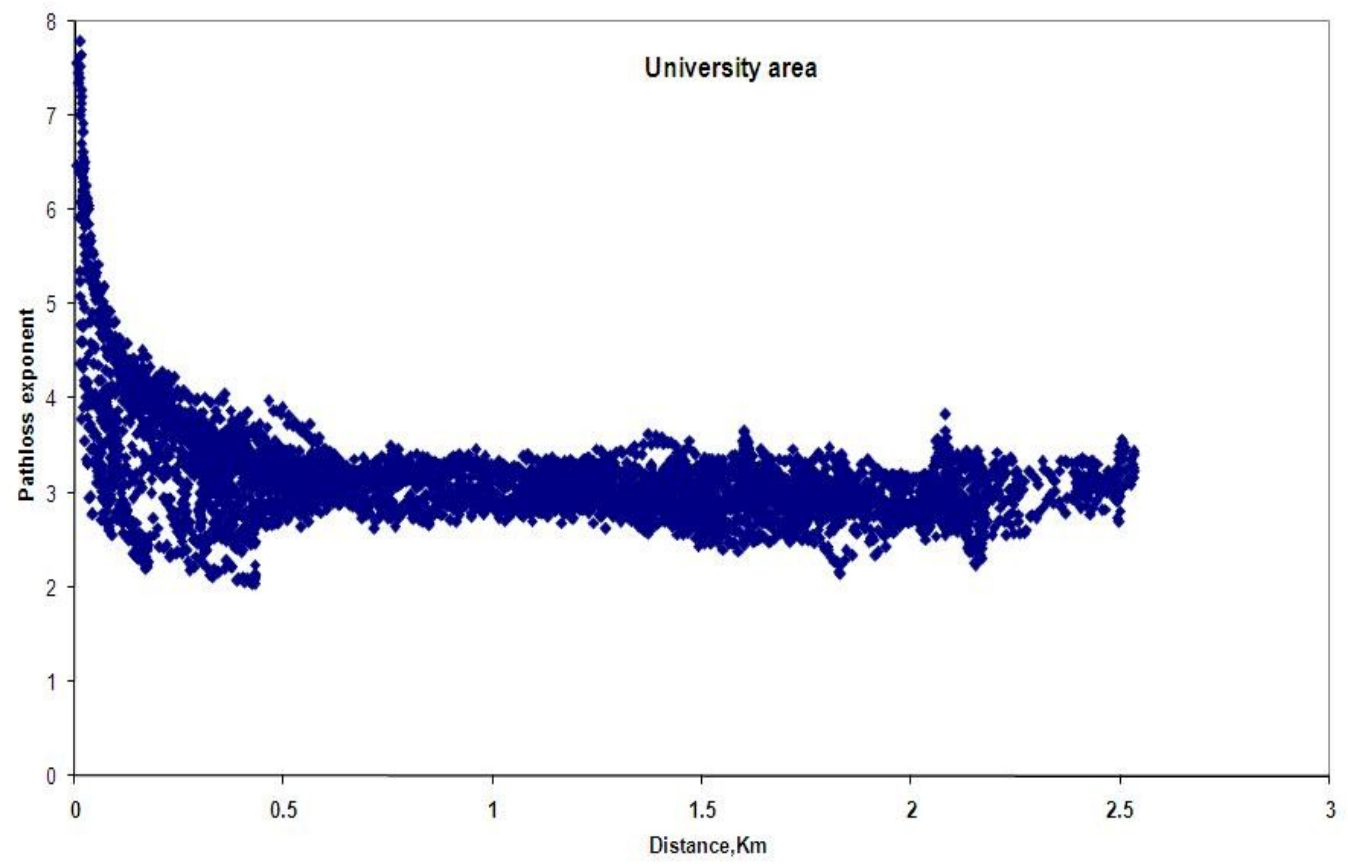

Fig 9. Comparison of observed path losses with those deduced from various prediction methods for PVR base station

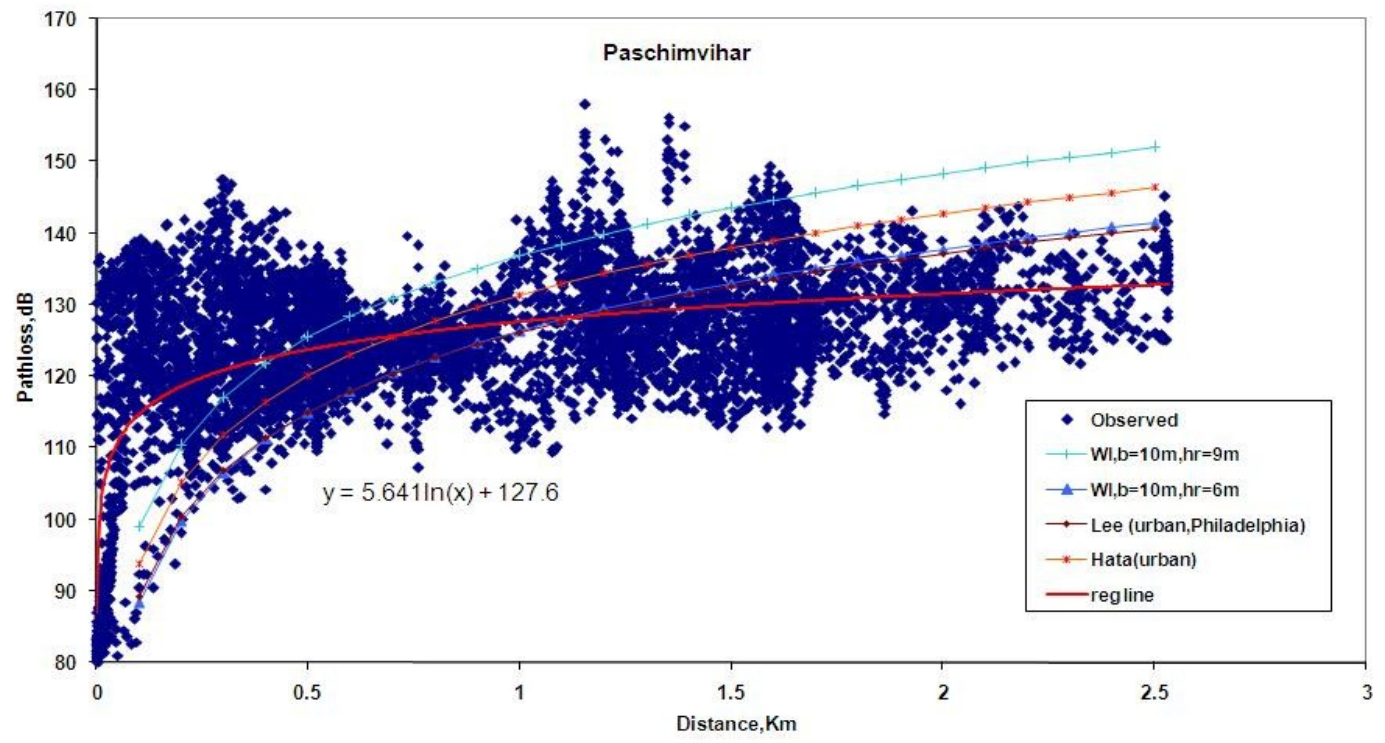


Fig10. Variation of path loss exponent as a function of distance for PVR base station

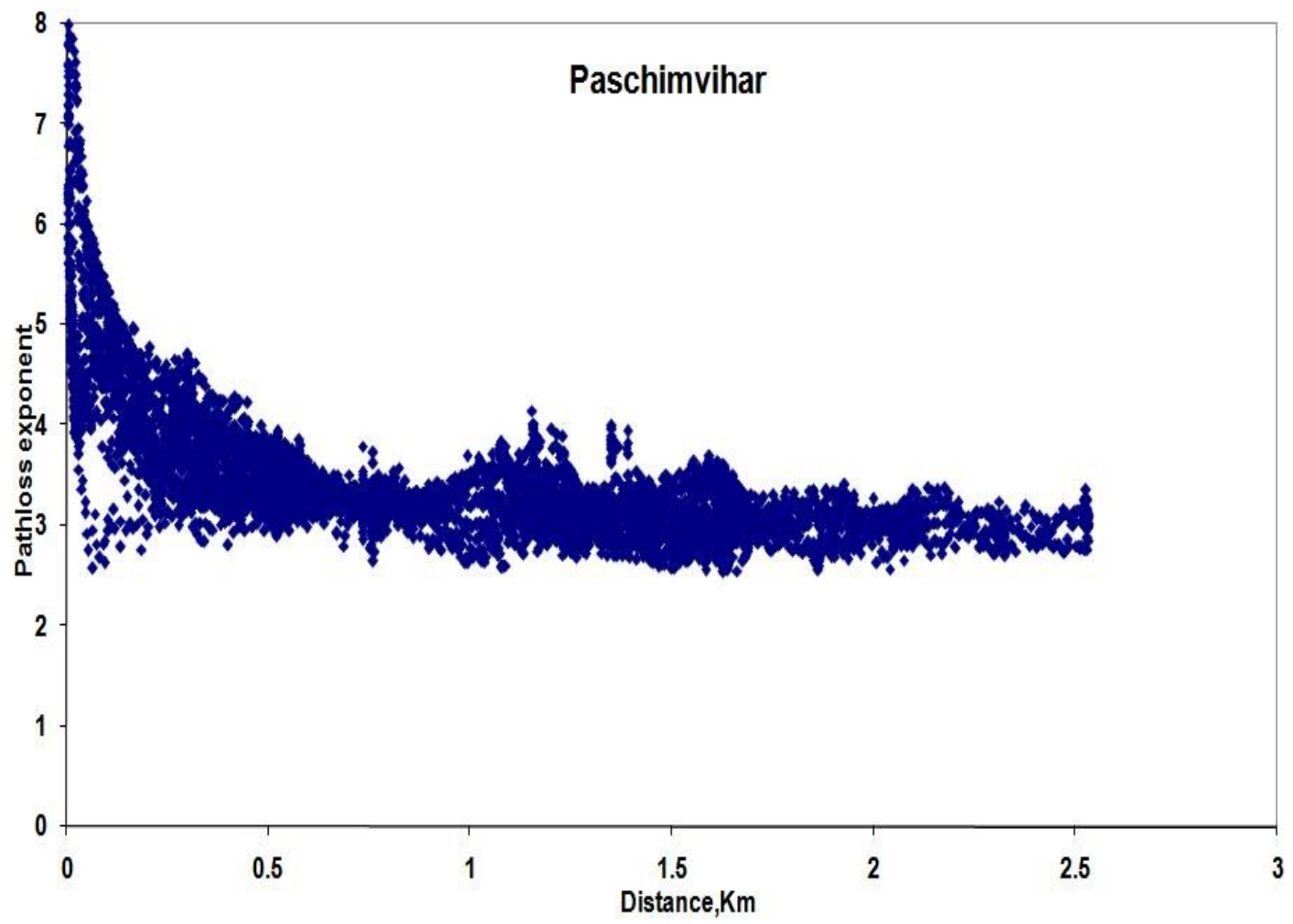

